\title{
Análisis a los estados financieros de empresas camaroneras en la provincia de El Oro, Ecuador
}

\section{Analysis of the financial statements of shrimp companies in the province of El Oro, Ecuador}

Ing. Juan Carlos Muñoz Briones, M.Sc.

Universidad Metropolitana, Ecuador

Autor para correspondencia: cjmunoz@umet.edu.ec ; jlibra_88@hotmail.com

Fecha de recepción: 23 de junio de 2018 - Fecha de aceptación: 30 de agosto de 2018

Resumen: Esta investigación, está enfocada al análisis de la situación económica según resultados de los estados financieros del sector camaronero en los cantones Huaquillas, Arenillas y Santa Rosa de la provincia de El Oro, durante los últimos años, con el propósito de determinar sus indicadores financieros como son liquidez, endeudamiento y rentabilidad, así como también evaluando su capital de trabajo; la metodología desarrollada en esta investigación es de tipo descriptiva, mediante el uso de un diseño no experimental de tipo longitudinal, cuyos resultados relevantes dentro del proceso de la investigación es que el sector camaronero vive uno de sus años de mayores auge, por sus avances tecnológicos, el dominio de conocimientos, el incremento en su producción y la permanencia de su más alta calidad, esto trae como consecuencia que exista una mayor demanda en el mercado nacional e internacional, para determinar las variables se usó la correlación de Pearson con la finalidad de determinar la relación entre variables analizadas, como son utilidad, deuda e inversión, además del análisis de las cuentas de activos, pasivos, patrimonio, ingresos, y costos/gastos, que permiten determinar la situación real de las empresas, coadyuvando a los directivos al entendimiento de sus resultados y fortaleciendo a la sostenibilidad al tomar decisiones idóneas en relación a los resultados razonables.

Palabras clave: Estados financieros, Liquidez, Rentabilidad, Análisis, toma de decisiones, sostenibilidad.

Abstract: This research is focused on the analysis of the economic situation according to results of the financial statements of the shrimp sector in the Huaquillas, Arenillas and Santa Rosa cantons of the province of El Oro, in recent years, in order to determine its financial indicators as are liquidity, indebtedness and profitability, as well as evaluating their working capital; the methodology developed in this research is descriptive, using a non-experimental design of longitudinal type, whose relevant results within the research process is that the shrimp sector lives one of its greatest boom years, due to its technological advances, the knowledge domain, the increase in its production and the permanence of its highest quality, this brings with it that there is a greater demand in the national and international market, to determine the variables Pearson correlation was used with the purpose of to determine the relationship between variables analyzed, such as utility, debt and investment, in addition to the analysis of the accounts of assets, liabilities, equity, income, and costs / expenses, which allow the real situation of companies to be determined, understanding of their results and strengthening sustainability by making appropriate decisions in relation to results reasonable.

Key Words: Financial statements, Liquidity, Profitability, Analysis, Indebtedness, Producers, Boom. 


\section{Introducción}

El sector camaronero a nivel nacional representa un rubro importante en la economía del Ecuador, según Banco Central (2016), las exportaciones de este producto representan en promedio 2400 millones de dólares, ubicándose en segundo lugar de los productos no petroleros, es superada por la exportación de banano que para este período representó 2500 millones. Llevar a cabo la presente investigación es relevante para tener conocimiento sobre el destino de las utilidades que tienen las empresas camaroneras, hacia las inversiones que éstas necesitan para poder ser más competitivos a nivel internacional, según Pro Ecuador (2016), Ecuador ocupa el primer lugar con un ranking de 4,6 en cuanto a mejor calidad de infraestructura, comparado con países como: Brasil, Costa Rica, Colombia y Guatemala siendo este último el segundo mejor con una calificación de 4,0.

El tema desarrollado se justifica en la necesidad de brindarles a las empresas camaroneras de la provincia de El Oro un análisis técnico basado en sus estados financieros en el mismo que se puede observar el comportamiento de sus utilidades y en qué porcentaje éstas se reinvirtieron en activos propios de su giro de negocio, de acuerdo a los datos proporcionados por la Dirección de Inteligencia Comercial e Inversiones (Pro Ecuador, 2016), el Ecuador ocupa el segundo lugar en eficiencia de los costos de operación e inversión, representando para ese período en promedio 3.400.000, lo cual representa un 7\% menos en relación a los demás países, significando que en el país es conveniente invertir en este sector ya que es menos costoso realizarlo, a diferencia de los países citados en el párrafo anterior.

La industria camaronera aporta aproximadamente con la generación de 180.000 plazas de empleo directo e indirecto, lo cual se suma en conjunto con la pesca hace que ascienda al $5 \%$ de las plazas de empleo del país. Estas oportunidades se generan en al menos 3000 fincas que ocupan al menos 210.000 hectáreas de extensión en las provincias costeras ecuatorianas. Así mismo los datos reportan que existen unas 277 embarcaciones dedicadas a la pesca con lo cual también se dinamiza el comercio. (Cámara de Acuacultura, CNA, 2017). Para el presidente de la Cámara Nacional de Acuacultura (CNA, 2017), José Antonio Campuzano, el valor agregado en productos como el camarón no tiene que ver necesariamente con la industrialización, sino con aspectos fundamentales, como la cría de larvas, su alimentación y el desarrollo tecnológico que ocurre desde las piscinas.

El objetivo general es realizar un análisis a los estados financieros de empresas camaroneras de la Provincia de El Oro. Y los específicos son analizar financieramente el balance general de las empresas camaroneras de la Provincia de El Oro, analizar financieramente el estado de resultados de las empresas camaroneras de la Provincia de El Oro.e identificar el grado de correlación entre las variables utilidad/inversión, deuda/inversión.

\section{Marco Teórico}

\section{Los Estados Financieros de la empresa y su análisis}

Los estados financieros son informes que se utilizan dentro de las empresas o instituciones para dar a conocer a los interesados la situación económica y financiera, además de mostrar los cambios que la empresa ha experimentado dentro de un determinado tiempo o periodo. Esta 
información es de gran utilidad para la gerencia porque le permite tener una visión global de la situación de la entidad. (Berk, DeMarzo, \& Hardford, 2010)

Según el marco conceptual de las NIIF se establece que el conjunto de estados financieros estará conformado por:

$\checkmark \quad$ El balance de situación o Estado de situación financiera, La cuenta de pérdidas y ganancias o Estado de Resultados Integrales, Estado de cambios en el patrimonio neto, Estado de flujos de tesorería o de efectivo y Notas aclaratorias o anexos. Los mismos que tiene que ser subidos en la plataforma virtual del organismo de control como es la Superintendencia de Compañías.

\section{El balance de Situación Financiera}

Es un informe contable que presenta ordenada y sistemáticamente las cuentas de Activos, Pasivos y Patrimonio, y determina la posición financiera de la empresa en un momento determinado.

Las partes y formas de presentación del estado de situación financiera son:

- Encabezamiento. Contendrá nombre o razón social, número de identificación, periodo al que corresponde la información, nombre del estado, cierre del balance y denominación de la moneda que se presenta.

- Texto. Es la parte esencial que presenta las cuentas de Activo, Pasivo y Patrimonio distribuidas de tal manera que permitan efectuar un análisis financiero objetivo.

- Firmas de Legalización. En la parte inferior del Estado se deberán insertar la firma y rúbrica del contador y gerente. (Zapata, 2011)

\section{Estado de Resultados Integrales o Estado de pérdidas y ganancias}

El estado de resultados mide el desempeño durante un periodo específico, por ejemplo, un año. La definición contable de utilidad es: Ingresos - gastos = utilidades. La sección de operaciones registra los ingresos y gastos de la empresa provenientes de las operaciones principales. Una cifra de particular importancia es la de utilidades antes de impuestos que resume las utilidades antes de impuestos y costos de financiamiento. Entre otras cosas, la sección no operativa del estado de resultados incluye todos los costos de financiamiento, como los gastos por intereses. (Ross, Westerfield, \& Jaffe, 2012)

\section{Estados de Cambios en el Patrimonio}

El estado de situación patrimonial refleja la situación del patrimonio de la empresa en un momento determinado, desglosa los fondos propios calculados en el balance de los fondos que provienen de la emisión de nuevas acciones frente a los beneficios no disponibles. Dado que el valor contable patrimonial no es una valoración útil para fines financieros. (Berk, De Marzo, \& Hardford, 2010) 


\section{Estado de Flujo de Efectivo}

Estado contable que muestra cuáles han sido las principales fuentes de dinero de la empresa y como las ha utilizado durante un periodo determinado. Actividades de explotación: empieza con el beneficio neto de la cuenta de resultados, luego se ajusta esta cifra añadiendo que no suponen ingresos efectivos relacionados con las actividades de explotación de la empresa. Actividades de inversión: enumera el efectivo usado en inversiones. Actividades financieras: Muestra el flujo de efectivo entre la empresa y sus inversores. Este estado ayuda a explicar los cambios en el efectivo contable. (Ross, Westerfield, \& Jaffe, 2012)

\section{Técnicas y herramientas de análisis financieros}

(Ferrer, 2012) Menciona que en el análisis de los estados financieros deben existir proporciones, adecuadas o no, entre las distintas partidas integrantes de estos, las cuales ponen a disposición nuevos elementos de juicio para el estudio de la situación financiera de la entidad.

\section{Análisis de las razones o ratios financieros}

\section{Razones de Liquidez}

La liquidez se refiere a la facilidad y rapidez con que los activos se pueden convertir en efectivo. El activo circulante, incluye el efectivo y los activos que se convertirán en efectivo en el trascurso de un año a partir de la fecha del balance general. El inventario se compone de las materias primas que se usarán en la producción, el trabajo en proceso y los artículos terminados. (Ross, Westerfield, \& Jaffe, 2012)

Tabla 1: Razones de liquidez

\begin{tabular}{|l|l|l|}
\hline \multicolumn{2}{|c|}{ Razones de Liquidez } \\
\hline \hline 1 & Razón Corriente & Activo corriente/ pasivo corriente \\
\hline 2 & Prueba ácida & (Activo corriente - Inventario) /pasivo corriente \\
\hline 3 & Razón de efectivo & Efectivo / pasivo corriente \\
\hline 4 & Capital neto de trabajo & Activo corriente - pasivo corriente \\
\hline
\end{tabular}

\section{Razones de Actividad}

Se emplean para medir la velocidad a la que diversas cuentas que maneja la empresa se convierten en ventas o en efectivo. Entre las que se encuentra la siguiente clasificación con sus respectivas fórmulas de aplicación.

Tabla 2: Razones de actividad 


\begin{tabular}{|l|l|l|}
\hline \multicolumn{2}{|c|}{ Razones de Actividad } \\
\hline \hline 1 & Rotación del activo total & Ventas/activo total \\
\hline 2 & Rotación del activo fijo & Ventas/ activo fijo \\
\hline 3 & Rotación del inventario & Costo de ventas/ inventario \\
\hline 4 & Rotación de las cuentas por cobrar & Ventas a crédito /cuentas por cobrar \\
\hline 5 & Periodo promedio de cobro & 365 x cuentas por cobrar/ ventas \\
\hline 6 & Rotación de las cuentas por pagar & Compras a crédito /cuentas por pagar \\
\hline 7 & Periodo promedio de pago & 365 x cuentas por pagar /compras \\
\hline
\end{tabular}

\section{Razones de Endeudamiento}

Es un coeficiente usado para valorar el apalancamiento de las empresas, que se calcula dividiendo el importe total de la deuda a corto y largo plazo. Por el patrimonio neto o fondos propios, que se puede calcular sobre la base del valor de mercado o del valor contable. (Berk, DeMarzo, \& Hardford, 2010)

Tabla 3: Razones de apalancamiento

\begin{tabular}{|r|l|l|}
\hline \multicolumn{2}{|c|}{ Razones de Apalancamiento } \\
\hline \hline 1 & Razón de endeudamiento & Pasivo total/ activo total \\
\hline 2 & Índice de de capital & Pasivo a largo plazo/ capital \\
\hline 3 & Impacto de la carga financiera & Gastos financieros /ventas neetas \\
\hline 4 & Endeudamiento a corto plazo & Pasivo corriente/ pasivo total \\
\hline 5 & Apalancamiento a corto plazo & Pasivo corriente /Patrimonio \\
\hline
\end{tabular}

\section{Razones de Rentabilidad}

Son utilizadas por los analistas financieros para determinar si las empresas son capaces de utilizar eficientemente sus activos. Estima, además los resultados de utilidad de la entidad con relación a las ventas, activos o de inversión del propietario.

\section{Tabla 4: Razones de rentabilidad}

\begin{tabular}{|r|l|l|}
\hline \multicolumn{2}{|c|}{ Razones de Rentabilidad } \\
\hline \hline 1 & Margen neto de utilidad & Utilidad neta después de impuestos/ventas \\
\hline 2 & Margen bruo de utilidad & Utilidad bruta antes de impuestos /ventas \\
\hline 3 & Rendimiento del activo total & Utilidad neta después de impuestos/activo total \\
\hline 4 & Rendimiento del capital & Utilidad neta después de impuestos/ capital \\
\hline
\end{tabular}

\section{Método}

La presente Investigación es un diseño no experimental de tipo longitudinal, de evolución de grupo para un análisis comparativo. La información considerada para los respectivos análisis se encuentra evidenciada en los estados financieros de las empresas camaroneras, que mediante la investigación histórica y descriptiva se pudo conocer su situación económica como la evolución de las inversiones en relación a su utilidad, realizadas por este tipo de empresas. Se 
hizo uso de la correlación para identificar el grado de relación entre variables: utilidad/inversión, deuda/inversión. Con el fin de dar a conocer la razonabilidad de la economía de la empresa y poder determinar decisiones coherentes ante la situación enmarcando a la sostenibilidad de la misma.

\section{Resultados}

\section{Como resultado a los Análisis de indicadores financieros se determina lo siguiente:}

Tabla 5: Razones de liquidez

\begin{tabular}{|c|c|c|c|c|}
\hline Indicador & Fórmula & & 2015 & 2016 \\
\hline Razón Corriente & Activo corriente / Pasivo Corriente & $\$$ & 1,13 & 1,58 \\
\hline Prueba ácida & Activo corriente - Inventario/ Pasivo Corriente & $\$$ & 0,36 & 0,19 \\
\hline Capital neto de trabajo & Activo Corriente - Pasivo Corriente & $\$$ & $59.576,15$ & $\$ 214.439,78$ \\
\hline
\end{tabular}

Fuente: Anexo A.

Los resultados obtenidos durante los periodos de revisión el sector camaronero se demuestra que tienen un capital de trabajo lo suficientemente solvente. Ambos periodos denotan la fuerte incidencia del nivel de inventarios dentro del global de activos circulantes, esto a razón de la disminución de los inventarios en el activo circulante en el 2016 dando como resultado la irrisoria cantidad de 0,19 centavos por cada dólar de deuda, para lo cual espera equiparar la diferencia a través de la rotación de inventarios.

Tabla 6: Razones de endeudamiento

\begin{tabular}{|c|c|c|c|c|c|}
\hline Indicador & Fórmula & \multicolumn{2}{|c|}{2015} & \multicolumn{2}{|c|}{2016} \\
\hline Nivel de endeudamiento & Total Pasivo/Total Activo & $\$$ & 0,62 & $\$$ & 0,46 \\
\hline $\begin{array}{c}\text { Concentración del } \\
\text { endeudamiento a corto } \\
\text { plazo }\end{array}$ & Pasivo Corriente/ Pasivo Total & $\$$ & 1,00 & $\$$ & 1,00 \\
\hline Apalancamiento & Pasivo Corriente /Patrimonio & $\$$ & 1,63 & $\$$ & 0,85 \\
\hline $\begin{array}{c}\text { Impacto de la carga } \\
\text { financiera }\end{array}$ & Gastos Financieros/Ventas & & $9 \%$ & & $8 \%$ \\
\hline
\end{tabular}

Fuente: Anexo A.

Demostrando que en el periodo 2015 el nivel de endeudamiento según el indicador representa que el sector camaronero por cada dólar de inversión en activos cuenta \$0,62 financiados por sus entidades financieras o por sus acreedores, en el periodo 2016 esta relación disminuyó a los \$0,46, debido a la disminución del pasivo corriente y el aumento significativo del activo corriente.

El indicador de concentración del endeudamiento a corto plazo no ha sufrido variación debido a que el sector cataloga a sus deudas bancarias o de proveedores como activos corrientes, careciendo de pasivos no corrientes, dando un indicador de $100 \%$, representando que el total de sus activos están comprometidos con agentes externos.

El nivel de apalancamiento mide que tan comprometido está el patrimonio en relación con las deudas, es decir que por cada $\$ 1$ de Patrimonio cuanto se tiene de deuda para ese año, en 
el 2015 se tuvo $\$ 1,63$, mientras que para el $2016 \$ 0,85$.

El indicador financiero de impacto mide el grado de incidencia de los gastos financieros en relación a las ventas del periodo, en el año 2015 representaron el 2,69\%, mientras que para el 2016 se redujo a $2,08 \%$.

Tabla 7: Razones de actividad

\begin{tabular}{|c|c|c|c|c|}
\hline Indicador & Fórmula & 2015 & 2016 & Forma \\
\hline $\begin{array}{c}\text { Rotación de } \\
\text { Cartera }\end{array}$ & $\begin{array}{c}\text { Ventas a crédito en el periodo/Cuentas por } \\
\text { cobrar promedio }\end{array}$ & 15,39 & 20,39 & Veces \\
\hline $\begin{array}{l}\text { Ciclo de } \\
\text { cobro }\end{array}$ & $\begin{array}{c}\text { (Cuentas por cobrar promedio x } 365 \\
\text { días)/Ventas a crédito }\end{array}$ & 23,72 & 17,90 & Días \\
\hline $\begin{array}{l}\text { Rotación de } \\
\text { inventario }\end{array}$ & Costo de ventas/ Inventario promedio & 3,21 & 1,49 & Veces \\
\hline $\begin{array}{c}\text { Ciclo de } \\
\text { inventarios } \\
\text { días }\end{array}$ & Costo de ventas/ Inventario promedio & 113,71 & 244,97 & Días \\
\hline $\begin{array}{c}\text { Rotación de } \\
\text { los activos } \\
\text { fijos }\end{array}$ & Ventas/ Activo fijo bruto & 6,18 & 4,85 & Veces \\
\hline $\begin{array}{c}\text { Rotación de } \\
\text { activos } \\
\text { totales }\end{array}$ & Ventas/ Activos totales & 1,83 & 1,30 & Veces \\
\hline $\begin{array}{c}\text { Rotación de } \\
\text { cuentas por } \\
\text { pagar }\end{array}$ & Compras anuales a crédito/ Proveedores & 11,11 & 8,35 & Veces \\
\hline $\begin{array}{c}\text { Ciclo de } \\
\text { cuentas por } \\
\text { pagar en días }\end{array}$ & 365 días/ Rotación de cuentas por pagar & 32,84 & 43,73 & Días \\
\hline $\begin{array}{l}\text { Ciclo del } \\
\text { efectivo }\end{array}$ & $\begin{array}{c}\text { Ciclo de inventario + ciclo de cobro - ciclo } \\
\text { de pago }\end{array}$ & 104,59 & 219,14 & Días \\
\hline
\end{tabular}

Fuente: Anexo A Y B.

\section{Análisis:}

El índice de rotación de cartera refleja las veces en las que las cuentas por cobrar del sector han rotado con relación a las ventas, en el año 2015 rotaron 15, 39 ocasiones, mientras que en el año 2016 las cuentas por cobrar rotaron en 20,39 ocasiones, este incremento se puede reconocer debido a la disminución tanto de las ventas como de las cuentas por cobrar en el periodo 2016. Existen diversas formas de calcular las rotaciones de las cuentas por cobrar, una que analizamos fue el ciclo de cobro en la que se definen los días en los que rota las cuentas por cobrar, en el año 2015 las cuentas por cobrar rotaron cada 24 días, mientras que en el 2016 rotaron cada 18 días aproximadamente.

El indicador de rotación de inventarios como su nombre lo define, muestra las veces en las que el inventario rota dentro de un periodo, en el 2015 los inventarios rotaron 3,21 veces en el año, es decir cada 114 días, mientras que en el 2016 los inventarios rotaron 1,49 veces, es decir cada 245 días, esta disminución en la rotación del inventario fue ocasionada por el aumento en la cantidad de inventario en el periodo y la disminución del Costo de Producción y ventas. 
El indicador de rotación de activos fijos refleja que los activos fijos rotaron en 6,18 ocasiones en el 2015, mientras que en el año 2016 rotaron en 4,85 ocasiones. Lo que significa que por cada dólar invertido en activos fijos se obtuvo $\$ 6,18$ en el 2015 y en el 2016 se obtuvo $\$ 4,85$.

El indicador de rotación de los activos totales refleja que los activos totales rotaron 1,83 veces en el 2015, mientras que en el 2016 rotaron 1,30 veces, esto debido a la disminución en el nivel de ventas. Además de ello se determina que por cada dólar invertido en los activos totales se generó \$1,83 en el 2015 y \$1,30 en el 2016. El nivel de rotación de cuentas por pagar durante el año 2015 fue de 11,11; mientras que en el 2016 fue de 8,35 ocasiones, esto significa que el sector camaronero pagaba a sus proveedores en el 2015 cada 33 días y cada 8 días en el 2016. El indicador de ciclo de efectivo muestra que el sector camaronero debió esperar 105 días en el año 2015 para que el efectivo utilizado en el proceso productivo retorne, mientras que en el 2016 transcurrieron 219 días.

Tabla 8: Razones de rentabilidad

\begin{tabular}{|c|c|c|c|}
\hline Indicador & Fórmula & $\mathbf{2 0 1 5}$ & $\mathbf{2 0 1 6}$ \\
\hline \hline Margen Bruto & Utilidad bruta/ Ventas & $16 \%$ & $42 \%$ \\
\hline Margen Neto & Utilidad neta/ Ventas & $0,08 \%$ & $3,79 \%$ \\
\hline $\begin{array}{c}\text { Rendimiento del } \\
\text { Patrimonio }\end{array}$ & Utilidad neta/ Patrimonio & $0,41 \%$ & $26,94 \%$ \\
\hline $\begin{array}{c}\text { Rendimiento del } \\
\text { Activo Total }\end{array}$ & Utilidad neta/ Activo total & $0,16 \%$ & $14,48 \%$ \\
\hline
\end{tabular}

Fuente: Anexo A Y B.

La razón que muestra el margen bruto significa que las ventas del sector camaronero generaron un $0,08 \%$ de utilidad en el 2015, mientras que en el 2016 se determinó un margen de $42 \%$, por lo que se puede explicar en términos monetarios también debido a que por cada dólar de ventas generó 0,42 centavos de utilidad en el año 2016.

El indicador de margen operacional muestra que la utilidad operacional en relación a las ventas en el 2015 representa un 8\%, mientras que en el 2016 representa un 3,79\%, este incremento en el indicador para este año es el reflejo del aumento de los ingresos, que no se vio afectada por haber incurrido en mayores costos de producción y ventas. En términos monetarios en el 2015 por cada dólar de ventas se obtuvo \$0,08 centavos y en el 2016 se obtuvo \$3,79 dólares de utilidad operacional.

El Indicador del rendimiento del patrimonio muestra que las utilidades netas equivalen al $0,41 \%$ del patrimonio en el 2015, en el periodo del 2016 equivale a $26,94 \%$, este indicador indica además que se obtuvo un rendimiento del 26,94\% en el 2016.

El indicador que mide el rendimiento del activo total en relación a la utilidad neta en el año 2015 representó un $0,16 \%$, y para el año 2016 fue de 14,48\%, lo que significa que la utilidad 
por cada dólar de inversión en los activos totales generó \$0,16 dólares, en el año 2016 fue de \$14,48 dólares de rentabilidad.

\section{Determinación de correlación entre Utilidad e Inversión}

Para la presente investigación se consideró necesario ver si existe o no un grado de relación entre las variables antes mencionadas, para así conocer cuál es el comportamiento ante un cambio significativo de una de las dos variables.

Tabla 9: Correlación: Utilidad/Inversión

\begin{tabular}{ccccccccc}
\hline Año & UTILIDA & INVERSIÓ & $\mathrm{x}^{\wedge} 2$ & $\mathrm{y}^{\wedge} 2$ & $\mathrm{xy}$ & $\mathrm{x}-\mathrm{X}$ & $\mathrm{y}-\mathrm{Y}$ & $(\mathrm{x}-\mathrm{X})(\mathrm{y}-\mathrm{Y})$ \\
& $\mathrm{D} \mathrm{X}$ & $\mathrm{N} \mathrm{Y}$ & & & & & & \\
\hline $\mathbf{2 0 1}$ & 1112,17 & 69214,11 & 1236922,10 & 479059302 & 76977856 & - & - & 210412851 \\
$\mathbf{5}$ & & & 9 & 3 & 72 & 56905,2498 & 3697, &, 8 \\
& & & & & 4 & 6 & \\
$\mathbf{2 0 1}$ & 114922,66 & 76609,31 & 132072200 & 586898637 & 880414642 & 56905,2498 & 3697, & 210412851 \\
$\mathbf{6}$ & 97 & & 06 & 9 & 8 & 4 & 6 &, 8 \\
$\Sigma$ & $\mathbf{1 1 6 0 3 4 , 8 3}$ & $\mathbf{1 4 5 8 2 3 , 4 2}$ & $\mathbf{1 3 2 0 8 4 5 6 9}$ & $\mathbf{1 0 6 5 9 5 7 9 4}$ & $\mathbf{8 8 8 1 1 2 4 2 8}$ & $\mathbf{0}$ & $\mathbf{0}$ & $\mathbf{4 2 0 8 2 5 7 0 3}$ \\
& $\mathbf{9 7}$ & & $\mathbf{2 8}$ & $\mathbf{0 2}$ & $\mathbf{4}$ & & & $\mathbf{6}$ \\
\hline
\end{tabular}

Fuente: Anexo A Y B.

\begin{tabular}{|r|c|}
\hline \multicolumn{2}{|c|}{ MEDIAS } \\
\hline $\boldsymbol{X}$ & 58017,41984 \\
\hline $\boldsymbol{Y}$ & 72911,71 \\
\hline
\end{tabular}

$S x=\sqrt{\frac{\sum_{x} 2-\frac{\left(\sum x\right)^{2}}{n}}{n-1}}=80476,17$

$$
S y=\sqrt{\frac{\sum_{y} 2-\frac{\left(\sum y\right)^{2}}{n}}{n-1}}=5229,19
$$

$\mathrm{r}=\frac{\sum(x-X)(y-Y)}{n-1(S x)(S y)}=\frac{420825703,6}{420825703,6}=1$ 


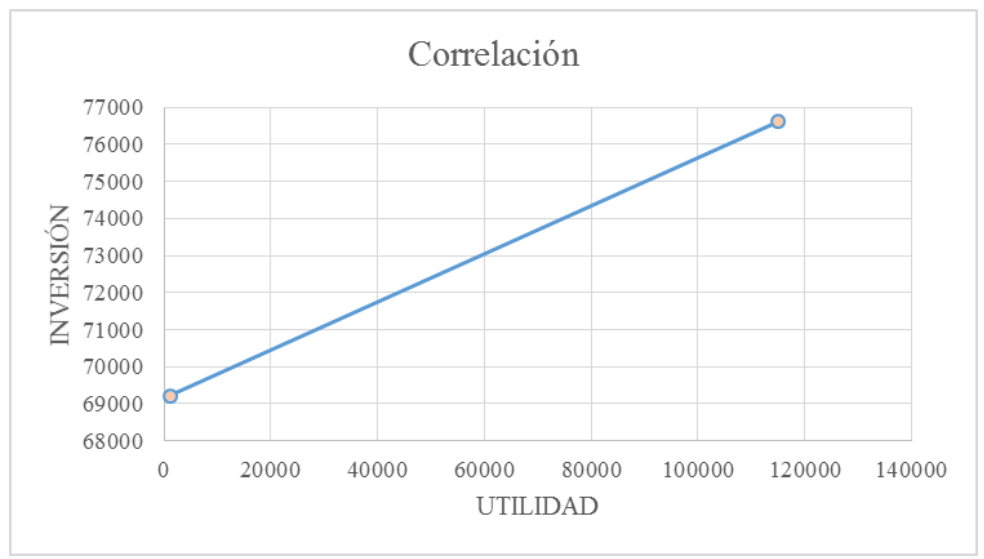

Figura 1: Correlación: Utilidad/Inversión Fuente: Anexo A Y B.

Como es de conocimiento correlación expresa el grado de relación que tiene una variable con respecto a otra, es aquí cuando mediante el coeficiente $r$ obtenido se puede analizar que sucede ante un cambio de una variable con la otra. Según el resultado $r=1$ si existe correlación (directa) entre la utilidad y la inversión, la misma que es positiva perfecta, indicando este valor que, si la variable independiente sufre un cambio, la dependiente puede sufrir un aumento en la misma proporción constante.

\section{Determinación de correlación entre Deuda e Inversión}

De igual forma se consideró necesario ver si existe o no un grado de relación entre las variables antes mencionadas, para así conocer cuál es el comportamiento ante un cambio significativo de una de las dos variables.

Tabla 10: Correlación: Deuda/Inversión

\begin{tabular}{|c|c|c|c|c|c|c|c|c|}
\hline $\mathrm{N}^{\circ}$ & DEUDA X & INVERSIÓN Y & $x^{\wedge} 2$ & $\mathrm{y}^{\wedge} 2$ & xy & $x-X$ & $y-Y$ & $\begin{array}{c}(x-X)(y- \\
Y)\end{array}$ \\
\hline 201 & & & 79413009 & 47905930 & 61679446 & - & - & 95268483 \\
\hline 5 & $89.113,98$ & $69.214,11$ & 86 & 23 & 41 & $\begin{array}{c}25764,951 \\
25\end{array}$ & $\begin{array}{c}3697, \\
6\end{array}$ & ,74 \\
\hline 201 & & & 19780700 & 58689863 & 10774630 & 25764,951 & 3697 , & 95268483 \\
\hline 6 & $140.643,88$ & $76.609,31$ & 981 & 79 & 603 & 25 & 6 & ,74 \\
\hline$\Sigma$ & & & 27722001 & 10659579 & 16942575 & $\mathbf{0}$ & $\mathbf{0}$ & 19053696 \\
\hline & $229.757,86$ & $145.823,42$ & 967 & 402 & 244 & & & 7,5 \\
\hline
\end{tabular}

\begin{tabular}{|r|c|}
\hline \multicolumn{2}{|c|}{ MEDIAS } \\
\hline $\boldsymbol{X}$ & 114878,93 \\
\hline $\boldsymbol{Y}$ & 72911,71 \\
\hline
\end{tabular}

$S x=\sqrt{\frac{\sum_{x} 2-\frac{\left(\sum x\right)^{2}}{n}}{n-1}}=36437,14$ 


$$
\begin{aligned}
& S y=\sqrt{\frac{\sum_{y} 2-\frac{\left(\sum y\right)^{2}}{n}}{n-1}}=5229,19 \\
& \mathrm{r}=\frac{\sum(x-X)(y-Y)}{n-1(S x)(S y)}=\frac{190536967,48}{190536967,48}=1
\end{aligned}
$$



Figura 2: Correlación: Deuda/Inversión

Fuente: Anexo A Y B.

Según el resultado $r=1$ si existe correlación entre la deuda y la inversión, la misma que es positiva perfecta, indicando este valor que, si la variable independiente sufre un cambio, la dependiente puede sufrir una variación en la misma proporción constante.

\section{Discusión}

\section{Conclusiones}

El sector camaronero de la Provincia de El Oro se encuentra financieramente fuerte y consolidado, dado que sus índices no muestran un eminente riesgo que pueda sufrir el sector, para el año 2016 registra pérdidas, esto se debe al declive en la economía a nivel mundial, contrastando que los países de Latinoamérica no se han visto muy afectados por ese suceso.

El sector camaronero de la Provincia de El Oro realiza inversiones constantes y éstas van destinadas hacia la adquisición de activos productivos, los mismos que son financiados con apalancamiento financiero, este es accesible debido a que el sector presenta utilidades en los diferentes períodos, conllevando todos estos aspectos positivos a determinar la existencia de un buen flujo de efectivo operacional.

\section{Bibliografía}

Aguado, J. (2005). Fundamentos de Inversiones (Segunda Edición ed.). Pearson Educación. 
Andino, P. (2014). Guía para elaboración de trabajos de titulación. Quito: UTE.

Baca, G. (2010). Evaluación de Proyectos. México: McGRAW-Hill/Interamericana Editores, S.A.

Baena, D. (2014). Análisis financiero enfoque y proyecciones. Bogotá: Ecoediciones.

Banco Central del Ecuador (2017) recuperado en https://www.bce.fin.ec/boletin504

Baptista, H. F. (2010). Metodología de la investigación. México: McGraw Hill.

Berk, J., DeMarzo, P., \& Hardford, J. (2010). Fundamentos de Finanzas Corporativas. Madrid: Pearson Education.

Cámara Nacional de Acuacultura (CNA, 2017) recuperado en http://www.cna ecuador.com/noticias camaronec.

Dirección de Inteligencia Comercial e Inversiones (Pro Ecuador, 2016)

Ehrhardt, M., \& Brigham, E. (2012). Finanzas Corporativas (Cuarta ed.). México: Cengage Learning.

Estupiñán, R. (2009). Estado de Flujos de efectivo y de otros flujos de fondos (Segunda ed.). Bogotá: Ecoediciones.

Ferrer, A. (2012). Estados financieros análisis e interpretaciones. Lima: Pacífico Ediciones.

Marques, M. (2015). Estadística básica: Un enfoque no paramétrico. México: Universidad Nacional Autónoma de México.

Piñeiro, C., \& de Llano, P. (2012). Finanzas Empresariales (Primera ed.). Santiago de Compostela: Andavira Editores.

Proecuador (2017). Recuperado en http://www.proecuador.gob.ec/exportaciones 17

Rincón, C. (2011). Presupuestos Empresariales (Primera ed.). Bogotá: Ecoediciones.

Rodriguez, L. (2012). Análisis de los Estados Financieros. México: McGraw Hill.

Ross, S., Westerfield, R., \& Jaffe, J. (2012). Finanzas Corporativas (Novena ed.). México: McGraw Hill. 
Stanley, G. (2015). Fundamentos de la administración financiera (Decimocuarta ed.). México: McGraw Hill.

Universidad de Texas (2008). ABC de la correlación y sus aplicaciones sociales. EEUU: Universidad de Texas.

Zamorano, E. (2014). Análisis Financiero para la toma de decisiones. México: Innova

Zapata, Pedro. (2011). Contabilidad General. Colombia, 9na edición, Mc -Graw Hill.

\section{Anexo A. Balance General}

\begin{tabular}{|c|c|c|}
\hline \multicolumn{3}{|c|}{$\begin{array}{c}\text { CAMARONERAS } \\
\text { BALANCE GENERAL }\end{array}$} \\
\hline \multirow{2}{*}{$\begin{array}{l}\text { ACTIVOS } \\
\text { CORRIENTE }\end{array}$} & 2015 & 2016 \\
\hline & \\
\hline Efectivo y Equivalente de Efectivo & $76.187,07$ & $18.560,45$ \\
\hline Cuentas por Cobrar & $85.349,50$ & $50.450,00$ \\
\hline Provisión Cuentas Incobrable & $(4.267,48)$ & 0 \\
\hline Inventarios & $342.560,25$ & $510.522,50$ \\
\hline Anticipo de Impuesto Renta & $4.010,59$ & $1.867,29$ \\
\hline Anticipo de Impuesto IVA & 628,82 & - \\
\hline Total Activos Corrientes & $504.468,75$ & $581.400,24$ \\
\hline NO CORRIENTES & \multirow{3}{*}{$\begin{array}{r}218.273,15 \\
(5.730,25) \\
\end{array}$} & \\
\hline Propiedad Planta y Equipo & & $218.273,15$ \\
\hline Deprec-Acumulada P,P,E, & & $(6.052,43)$ \\
\hline Total Activos No Corrientes & $212.542,90$ & $212.220,72$ \\
\hline TOTAL ACTIVOS & $717.011,65$ & $793.620,96$ \\
\hline PASIVO & \multirow[b]{3}{*}{$356.455,91$} & \\
\hline CORRIENTES & & \\
\hline Obligaciones financieras & & $281.287,76$ \\
\hline Proveedores & $88.436,69$ & $85.672,70$ \\
\hline Total Pasivos Corriente & $444.892,60$ & $366.960,46$ \\
\hline TOTAL PASIVOS & $444.892,60$ & $366.960,46$ \\
\hline PATRIMONIO & \multirow{3}{*}{$\begin{array}{r}271.006,88 \\
1.112,17 \\
\end{array}$} & \\
\hline Capital Social & & $311.737,83$ \\
\hline Utilidad Liquida Ejercicio & & $114.922,67$ \\
\hline \multirow{2}{*}{$\begin{array}{l}\text { Total Patrimonio } \\
\text { TOTAL PASIVO + PATRIMONIO }\end{array}$} & 272.119,05 & $426.660,50$ \\
\hline & $717.011,65$ & 793.620,96 \\
\hline
\end{tabular}




\section{Anexo B. Estado de pérdidas y ganancias}

\begin{tabular}{|c|c|c|}
\hline $\begin{array}{r}\text { CAMA } \\
\text { ESTADO DE PÉRI }\end{array}$ & NCIAS & \\
\hline & 2015 & 2016 \\
\hline Ingresos & & \\
\hline Ventas & $1.313 .406,46$ & $3.028 .675,58$ \\
\hline Total de ingresos & $1.313 .406,46$ & $3.028 .675,58$ \\
\hline Inventario Inicial & $458.759,00$ & $1.553 .684,00$ \\
\hline Compras & $982.912,70$ & $715.094,47$ \\
\hline (-) Inv. Final & $(342.560,25)$ & $(510.522,50)$ \\
\hline Costo de Producción y venta & $(1.099 .111,45)$ & $(1.758 .255,97)$ \\
\hline Utilidad Bruta en Ventas & $214.295,01$ & $1.270 .419,61$ \\
\hline $\begin{array}{l}\text { GASTOS OPERACIONALES } \\
\text { Gastos de Administración }\end{array}$ & & \\
\hline Sueldos & $28.609,13$ & $42.935,95$ \\
\hline Beneficios Sociales, no gravados de IESS & $3.174,83$ & $5.035,78$ \\
\hline Aporte a la Seguridad Social & $3.823,46$ & $7.133,58$ \\
\hline Honorarios profesionales y dietas & 10,20 & - \\
\hline Gastos de gestión & $1.174,95$ & $4.691,13$ \\
\hline Impuestos y contribuciones & 969,61 & 21,64 \\
\hline Mantenimiento y Reparaciones & $11.298,38$ & $30.882,83$ \\
\hline Suministros y Materiales & $104.021,62$ & $12.571,09$ \\
\hline Combustibles y Lubricantes & $1.860,78$ & $1.055,53$ \\
\hline Total & \begin{tabular}{|l|l|}
$154.942,96$ \\
\end{tabular} & $104.327,53$ \\
\hline Gastos de Venta & & \\
\hline Depreciación & 322,19 & 322,19 \\
\hline Promoción y publicidad & $1.805,37$ & $6.750,02$ \\
\hline Transporte & $2.967,39$ & $2.678,58$ \\
\hline Provisión cuentas incobrables & $4.267,48$ & - \\
\hline Seguros y reaseguros & 101,50 & - \\
\hline Gastos de viaje & 625,14 & $123.445,00$ \\
\hline Otros Gastos & $12.143,33$ & $838.207,07$ \\
\hline Total & $22.232,40$ & $971.402,86$ \\
\hline Gastos Financieros & & \\
\hline Interes bancarios & $35.375,07$ & $21.351,86$ \\
\hline Total & $35.375,07$ & $21.351,86$ \\
\hline TOTAL GASTOS OPERATIVOS & \begin{tabular}{|l|l|}
$212.550,43$ \\
\end{tabular} & $1.097 .082,25$ \\
\hline GASTOS NO OPERACIONALES & & \\
\hline UTILIDAD ANTES $15 \%$ Trabajadores & $\mathbf{1 . 7 4 4 , 5 8}$ & 173.337,36 \\
\hline 15\% Trabajadores & 261,69 & $26.000,60$ \\
\hline BASE PARA CÁLCULO DE IMP-RTA & $1.482,89$ & $147.336,76$ \\
\hline Impuesto a la Renta & 370,72 & $32.414,09$ \\
\hline TOTAL GASTOS NO OPERATIVOS & 632,41 & $58.414,69$ \\
\hline TOTAL GASTOS & $213.182,84$ & $1.155 .496,94$ \\
\hline Utilidad Líquida & $\mathbf{1 . 1 1 2 , 1 7}$ & $114.922,67$ \\
\hline
\end{tabular}

